\title{
LA MORBILIDAD HOSPITALARIA DE LA POBLACIÓN ESPAÑOLA
}

\author{
POR \\ FERMINA ROJO PÉREZ \\ GLORIA FERNÁNDEZ-MAYORALAS FERNÁNDEZ \\ $\mathrm{Y}$ \\ JOSÉ MANUEL ROJO ABUIN
}

\section{Introducción}

La necesidad de asistencia sanitaria es el mayor predictor del uso de servicios médicos y de hospitalización (Wan and Odell, 1981). En general, existen dos vías para medir esta necesidad: la percepción individual del estado de salud y la evaluación objetiva o profesional (Hulka and Wheat, 1985), si bien diferentes autores han demostrado relaciones significativas entre ambas valoraciones (Linn and Linn, 1980; Newbold et al., 1995).

Las medidas subjetivas aportan un importante valor intrínseco como indicadores de problemas y necesidades comunitarios. Diversos estudios sobre la valoración subjetiva de la salud entre la población española han señalado la existencia de una diferente autopercepción en función de la edad y del sexo, así como de otras características socioeconómicas, y concluyen que a mayor edad y menor nivel socioeconómico existe una tendencia más alta a autovalorarse con una salud regular o mala, de igual forma que son las mujeres las que in-

Fermina Rojo Pérez, Gloria Fernández-Mayoralas Fernández, y José Manuel Rojo Abuin, Consejo Superior de Investigaciones Científicas, Madrid.

Estudios Geográficos

Tomo LVII, n. ${ }^{\circ} 227$, abril-junio 1997 
forman un peor estado de salud (Abellán García, A. et al., 1992; Rojo Pérez, F. et al., 1992; Fdez. Mayoralas Fernández, G. et al., 1993); este tipo de análisis deriva, básicamente, de encuestas de salud.

Por su parte, las medidas objetivas ofrecen una mayor capacidad comparativa, siendo la más estrictamente comparable, dentro del continuo de salud, la mortalidad, pero el éxito contra la mayoría de las enfermedades mortales le ha hecho perder parte de su capacidad informativa (Hansluwka, 1985), debiendo recurrirse, en ocasiones a medidas basadas en la enfermedad.

La situación se complica al constatar que sólo una pequeña proporción de la morbilidad conduce a la hospitalización (morbilidad hospitalaria) y muchas de las condiciones que requieren ingreso hospitalario no están relacionadas con el fenómeno de la muerte (Keskimaki et al., 1995). De hecho, una parte significativa de la utilización sanitaria depende de las decisiones facultativas (Käkkinen, 1991) a través de lo que se conoce como sistema de referencia.

En definitiva, los indicadores subjetivos y objetivos son complementarios y no suficientes en sí mismos (Andrews, 1981) para ofrecer una visión completa del nivel de salud de una población y de su necesidad y consumo de servicios sanitarios. Así, mientras las encuestas de salud ofrecen información sobre población «sana» (no institucionalizada), las medidas objetivas basadas en opiniones facultativas se realizan sobre población que ha accedido a los servicios sanitarios y, por tanto, «enferma», habiendo sido incluso institucionalizada en el caso de la información sobre morbilidad hospitalaria.

Todas las fuentes, estadísticas de servicios sanitarios pero también encuestas de salud, confirman que la morbilidad total se incrementa con la edad y que, en cada edad, es mayor entre las mujeres que entre los hombres (Davies, 1985). En este sentido, diversos autores han puesto de manifiesto que el factor preponderante de la frecuentación hospitalaria es la edad, amén de otros factores demográficos, lo que influye, además, en la duración de la estancia y el tipo de servicio (Sourty-le-Guellec, M. J., 1986); la tasa de presencia en hospital evoluciona en función de la edad siguiendo una curva en forma de «J», con una mayor frecuentación de mujeres, de viudos y divorciados, de obreros y empleados frente a cuadros superiores e inter- 
medios, y de jubilados y parados frente a activos (Sourty-le-Guellec, M. J., 1986; Sermet, C., 1987; Sourty-le-Guellec, M. J., 1993).

Estos estudios abordan, asimismo, los diagnósticos más frecuentes y la asociación y combinación entre diagnósticos (Com-Ruelle, L. et al., 1995). De este modo, en Francia, por ejemplo, los principales diagnósticos se relacionan con enfermedades del aparato circulatorio, mentales, del aparato digestivo, lesiones traumáticas y envenenamientos y tumores (Com-Ruelle, L. et al., 1989), mientras que en EE.UU. los diagnósticos más prevalentes se asocian, también en primer lugar, con las enfermedades del sistema circulatorio, seguidas por las del sistema digestivo, neoplasias, respiratorias y accidentes (Davies, 1985).

A la luz de estos trabajos, en este artículo se analiza la morbilidad en España, a partir de los diagnósticos hospitalarios y desde una perspectiva demográfica. Se parte de considerar que las enfermedades tratadas en ámbito hospitalario no se distribuyen de forma homogénea entre la población, sino que se manifiestan diferencias según sus características demográficas ${ }^{1}$.

Se pretende, así, contribuir al conocimiento del riesgo demográfico de padecer enfermedad para, una vez establecido su diagnóstico e incidencia, poder establecer políticas adecuadas de prevención y promoción de la salud general de toda la población.

\section{Fuentes y Metodología}

El estudio presente se realiza a partir del Conjunto Minimo Básico de Datos (C.M.B.C.), registro continuo que pretende sustituir a la actual Encuesta de Morbilidad Hospitalaria del I.N.E. escrutando todos los enfermos ingresados y dados de alta en los hospitales del Sistema Nacional de Salud (Rojo y Fdez.-Mayoralas, 1996). Los ítems de cada alta hospitalaria, recabados en el Ministerio de Sanidad y Consumo, en forma de fichero informático ${ }^{2}$, han sido los siguientes: nú-

1 Un estudio espacial de la distribución de altas hospitalarias de acuerdo al sistema de referencia comarcal, provincial o nacional, así como de las patologías según el lugar de residencia de los enfermos dados de alta será objeto de próximas contribuciones.

2 Para el uso de esta información ha sido necesario obtener el permiso de la Agencia de Protección de Datos. 
mero de historia clínica (convenientemente anonimizado para impedir su asociación con el paciente real), fecha de nacimiento, sexo, código postal del domicilio, financiación de la hospitalización, fecha de ingreso, circunstancias del ingreso, servicio donde se ingresa, fecha de alta, circunstancias del alta, diagnóstico principal, otros diagnósticos y hospital, de los que se han utilizado sólo algunos para el propósito del presente estudio.

Este fichero ha sido tratado con el programa SAS versión 6.09, implementado en el C.T.I.-C.S.I.C.; consta de 2.535.471 altas hospitalarias, de las que se han excluido 1.398 por carecer de información sobre diagnósticos. A partir de las variables originales se ha obtenido la edad del paciente y los diagnósticos se han agrupado por categorías según la Clasificación Internacional de Enfermedades 9. ${ }^{a}$ Revisión, Modificación Clínica (CIE-9-MC) (M. ${ }^{\circ}$ Sanidad y Consumo, 1994) ${ }^{3}$. El período al que están referidos los datos es el año 1994 , que coincide con la fecha de alta hospitalaria; a partir de la fecha de alta y de la fecha de ingreso hospitalario se ha calculado la duración de la estancia en número de días.

Este estudio aborda la caracterización de la población por edad y sexo, que ha causado alta hospitalaria en 1994, esto es, quiénes son y cuánto tiempo han permanecido hospitalizados, cómo han entrado y cómo han salido y las patologías que les han sido diagnosticadas. Este análisis se realiza por medio de tablas de doble entrada, que permiten describir la frecuencia relativa de cada uno de los sucesos y su interrelación.

3 Estas categorías son: 1: Enfermedades infecciosas y parasitarias; 2: Neoplasias; 3: Enfermedades endocrinas, nutritivas y metabólicas y trastornos de la inmunidad; 4: Enfermedades de la sangre y de los órganos hematopoyéticos; 5 : Trastornos mentales; 6 : Enfermedades del sistema nervioso y de los órganos de los sentidos; 7: Enfermedades del sistema circulatorio; 8: Enfermedades del aparato respiratorio; 9: Enfermedades del aparato digestivo; 10: Enfermedades del aparato genito-urinario; 11: Complicaciones del embarazo, parto y puerperio; 12: Enfermedades de la piel y del tejido subcutáneo; 13: Enfermedades del sistema osteo-mioarticular y tejido conectivo; 14: Anomalías congénitas; 15: Ciertas enfermedades con origen en el periodo perinatal; 16: Síntomas, signos y estados mal definidos; 17: Lesiones y envenenamientos; V: Clasificación suplementaria de factores que influyen en el estado de salud y contacto con los servicios sanitarios; E: Clasificación suplementaria de causas externas de lesiones y envenenamiento. 
Descripción de los pacientes que han causado alta hospitalaria

A) Edad y sexo.-De cada cuatro personas que han sido dadas de alta en hospital en 1994, casi dos tenían 65 o más años de edad, y una sobre diez tenía 80 y más; esto supone que, de entre todos los hospitalizados, un tercio son personas de edad y tan sólo un $13 \%$ tienen menos de 16 años (Cuadro I).

CuAdro I

ESTRUCTURA POR SEXO Y EDAD DE LA POBLACIÓN QUE HA CAUSADO ALTA HOSPITALARIA (1994)

(EN \% SOBRE N)

\begin{tabular}{crrr}
\hline Edad & Varón & Mujer & Total \\
\hline $0-1$ & 5,5 & 3,7 & 4,5 \\
$2-15$ & 11,0 & 6,5 & 8,6 \\
$16-39$ & 19,0 & 38,4 & 29,3 \\
$40-64$ & 30,0 & 23,0 & 26,3 \\
$65-74$ & 19,7 & 13,0 & 16,2 \\
$75+$ & 14,8 & 15,4 & 15,1 \\
$\mathrm{~N}$ & 1.190 .461 & 1.340 .904 & 2.531 .365 \\
\hline
\end{tabular}

La edad media de la población que ha causado alta hospitalaria es de 45,3 años con una desviación típica de 25 años; los jóvenes hasta los 15 años tienen una edad media de 5 años y una desviación de 4,8, lo que manifiesta una alta concentración de población infantil. Por su lado, la población de 65 y más años tiene una edad media de 75,3 y una desviación de 7,2; la proporción de población mayor de 75 años en hospital se multiplica por tres con respecto al mismo grupo demográfico dentro del conjunto de la población española (que es de 5,6 \%).

El perfil por edad muestra que los tres grupos más consumidores de servicios hospitalarios coinciden con la población entre 0 y 1 año, entre los 16 y 39 y de 55 y más años, que se relaciona con el nacimiento, la población en edad fértil y la población mayor. 
Por sexo, 53 de cada 100 personas que han causado alta hospitalaria son mujeres; según edades anuales, las mujeres predominan sobre los varones entre los 18 y los 42 años y a partir de los 81 años, en clara referencia a la edad del embarazo y del parto y a la mayor proporción de mujeres en las edades altas de la pirámide (Figura 1).

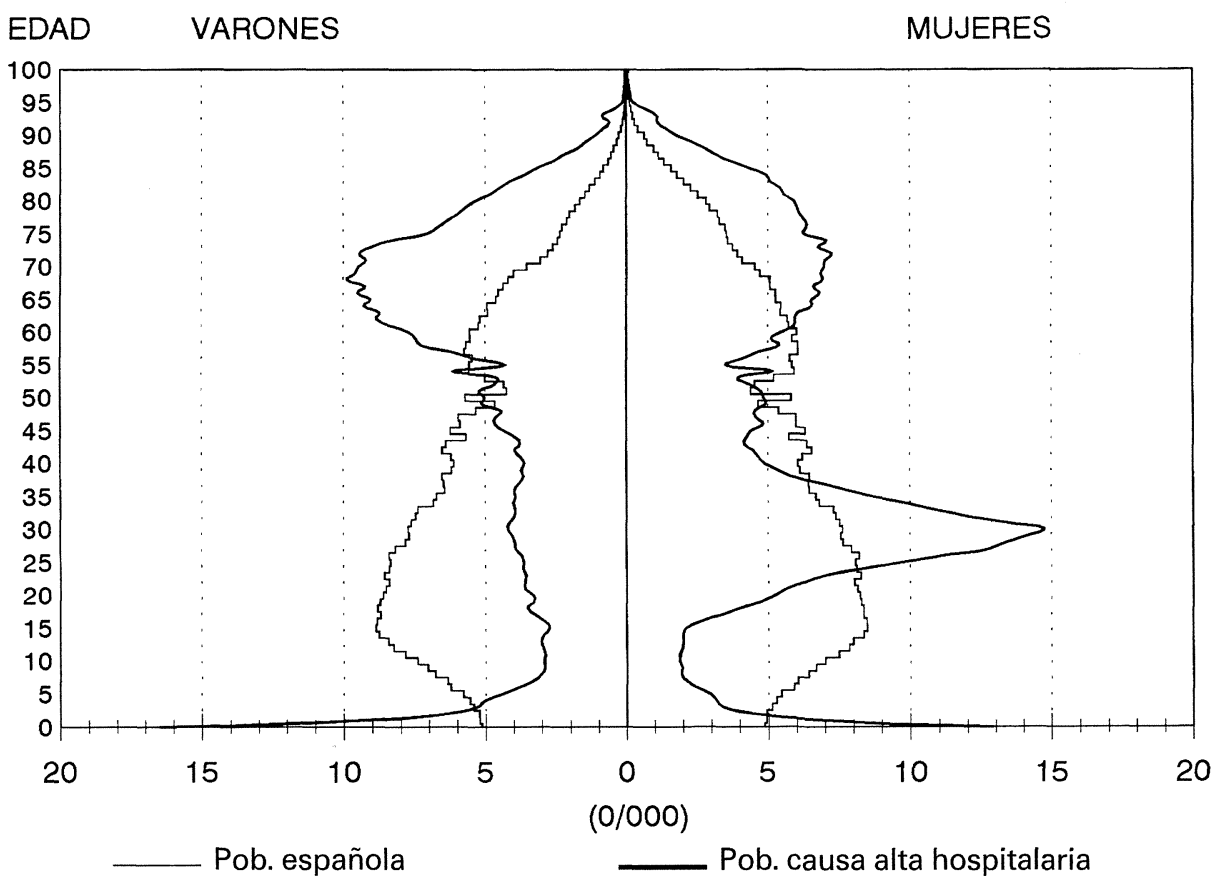

Figura 1.-Estructura de la población española (1991) y de la población que ha causado alta hospitalaria (1994) por sexo y edad anual.

Si se compara esta estructura de la población que ha causado alta hospitalaria con la de la población española en su conjunto, se constata que el predominio de mujeres en edad fértil en hospital es debido al fenómeno de maternidad, y que el predominio de mujeres a partir de los 80 años es un fenómeno demográfico.

B) Cómo han entrado (tipo de ingreso) y cómo han salido (circunstancias del alta).-Dos tercios de la población ha ingresado en hospital en 
1994 vía urgencia, siendo el conjunto de la población femenina, por un lado, y los ancianos, por otro, los más notables según este modo de ingreso (Cuadro II). Si se matiza por grupos de edad más pequeños, son los niños (0-1 años), los adultos jóvenes (entre 16-39 años) y las personas mayores (75 y más años) los que, generalmente, ingresan por urgencia.

\section{Cuadro II}

TIPO DE INGRESO DE LA POBLACIÓN QUE HA CAUSADO ALTA HOSPITALARIA (1994)

(EN \% SOBRE N)

\begin{tabular}{lccccccc}
\hline \multirow{2}{*}{$\begin{array}{c}\text { Tipo de } \\
\text { ingreso }\end{array}$} & \multicolumn{3}{c}{ Sexo } & & \multicolumn{2}{c}{ Edad } & \multicolumn{2}{c}{\begin{tabular}{c} 
Núm. medio \\
días \\
\cline { 2 - 7 }
\end{tabular}} & Varón & Mujer & Total & $\begin{array}{c}\mathbf{0 - 6 4} \\
\text { años }\end{array}$ & $\begin{array}{c}\mathbf{6 5}+ \\
\text { años }\end{array}$ & $\begin{array}{c}\text { Núm. medio } \\
\text { diagnós- } \\
\text { ticos }\end{array}$ \\
\hline Urgente & 59,4 & 63,6 & 61,6 & 60.5 & 64,1 & 9,3 & 2,4 \\
Programado & 40,6 & 36,4 & 38,4 & 39,5 & 35,9 & 7,0 & 1,8 \\
N & 1.187 .154 & 1.339 .049 & 2.526 .203 & 1.736 .353 & 789.850 & 8,5 & 2,1 \\
\hline
\end{tabular}

Los pacientes que acceden por urgencia al hospital suelen tener estancias más largas (media de 9,3 días) y mayor número de patologías diagnosticadas (media de 2.4 diagnósticos).

La mayoría de la población que abandona el hospital lo hace para regresar a su domicilio (Cuadro III). Las mujeres causan alta hospitalaria por regreso al domicilio en mayor proporción que los varones, mientras que éstos son trasladados a otro hospital o a un centro sociosanitario en mayor cuantía que las mujeres, y también se observa un tanto por ciento más importante de hombres que causan alta por motivo de muerte.

A medida que se envejece, se observa que la proporción de alta hospitalaria por traslado a domicilio disminuye y aumenta el traslado a otro hospital o centro sociosanitario, así como el alta por muerte.

C) Cuánto tiempo han permanecido en hospital.-La duración de la estancia en hospital ha sido de 8,5 días de media, llegando los ancianos a permanecer más de 11 . De este hecho deriva, pues, que el tramo más frecuente de estancia en hospital sea el de 1 a 7 días, que engloba al $60 \%$ de la población, mientras que en estancias largas o superiores a las 2 semanas permanece el $15 \%$ (Cuadro IV). 
Cuadro III

CIRCUNSTANCIAS DEL ALTA DE LA POBLACIÓN

QUE HA CAUSADO ALTA HOSPITALARIA (1994)

(EN \% SOBRE N)

\begin{tabular}{|c|c|c|c|c|c|}
\hline \multirow{2}{*}{$\begin{array}{c}\text { Circunstancias } \\
\text { del alta }\end{array}$} & \multicolumn{3}{|c|}{ Sexo } & \multicolumn{2}{|c|}{ Edad } \\
\hline & Varón & Mujer & Total & 0-64 años & 65+años \\
\hline A domicilio ....... & 91,5 & 93,6 & 92,6 & 94,6 & 88,2 \\
\hline \multicolumn{6}{|l|}{ Traslado a hospi- } \\
\hline tal $\ldots \ldots \ldots \ldots \ldots$ & 2,0 & 1,3 & 1,6 & 1,2 & 2,5 \\
\hline Alta voluntaria .. & 1,3 & 0,9 & 1,1 & 1,1 & 1,1 \\
\hline Exitus .............. & 3,4 & 2,3 & 2,8 & 1,3 & 6,1 \\
\hline \multicolumn{6}{|l|}{ Traslado a centro } \\
\hline sociosanitario & 0,5 & 0,4 & 0,5 & 0,3 & 0,9 \\
\hline Desconocido ..... & 1,3 & 1,5 & 1,4 & 1,5 & 1,2 \\
\hline $\mathrm{N}$ & 1.184 .690 & 1.333 .096 & 2.517 .786 & 1.729 .847 & 787.939 \\
\hline
\end{tabular}

\section{Cuadro IV}

DURACIÓN DE LA ESTANCIA DE LA POBLACIÓN QUE HA CAUSADO ALTA HOSPITALARIA (1994)

(EN \% SOBRE N)

\begin{tabular}{|c|c|c|c|c|c|}
\hline \multirow{2}{*}{$\begin{array}{l}\text { Días de } \\
\text { estancia }\end{array}$} & \multicolumn{3}{|c|}{ Sexo } & \multicolumn{2}{|c|}{ Edad } \\
\hline & Varón & Mujer & Total & 0-64 años & $65+a n ̃ o s$ \\
\hline 0 días .............. & 3,2 & 2,8 & 3,0 & 3,3 & 2,2 \\
\hline $1-7 \ldots \ldots \ldots \ldots \ldots \ldots$ & 56,3 & 64,3 & 60,5 & 68,0 & 44,0 \\
\hline 8-14 …............ & 22,9 & 20,0 & 21,4 & 17,9 & 29,0 \\
\hline $15-21 \quad \ldots \ldots \ldots \ldots \ldots$ & 8,7 & 6,6 & 7,6 & 5,4 & 12,5 \\
\hline $22-31 \quad \ldots \ldots \ldots \ldots \ldots$ & 4,9 & 3,5 & 4,2 & 2,9 & 6,9 \\
\hline $32+\ldots \ldots \ldots \ldots \ldots$ & 4,0 & 2,8 & 3,3 & 2,5 & 5,4 \\
\hline $\mathrm{N}$ & 1.190 .461 & 1.340 .904 & 2.531 .365 & 1.742 .141 & 791.974 \\
\hline Media .............. & 9,3 & 7,7 & 8,5 & 7,1 & 11,4 \\
\hline
\end{tabular}


Según el sexo, si bien hay más mujeres que hombres en hospital, éstos permanecen hospitalizados mayor número de días (9,3 días de media frente a 7,7), y la proporción de mujeres es superior a la de varones tan sólo en las estancias cortas (1 a 7 días).

Combinando las variables edad y sexo (Figura 2), destaca el hecho de que son los varones a partir de los 15 años los que permane-

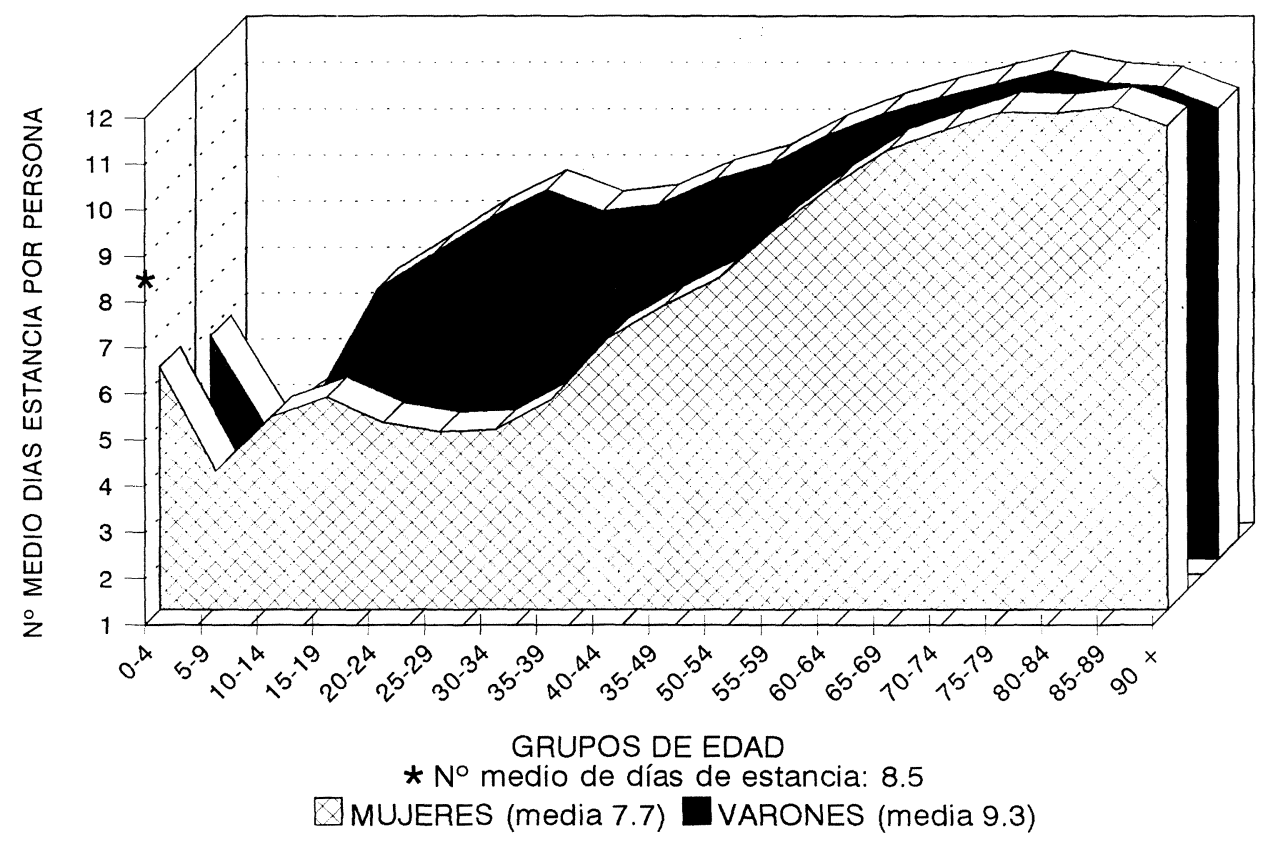

Figura 2.-Número medio de días de estancia en hospital de la población que ha causado alta hospitalaria (1994) por grupos de edad y sexo.

cen en hospital mayor número de días; así, en el grupo de población no anciana el factor maternidad no hace elevar la estancia hospitalaria, pues se observa que entre los 16 y 39 años el número medio de días de estancia hospitalaria es de 5 para las mujeres y de más del doble para los varones (13 días). Por el contrario, no se observan diferencias sustanciales en el subgrupo de los ancianos; las mujeres de 65 y más años manifiestan frecuencias sólo ligeramente superiores 
en las estancias largas, atribuibles, en todo caso, al factor demográfico, unido al padecer enfermedad crónica y degenerativa.

D) La enfermedad.-El criterio básico para justificar la estancia en hospital es la enfermedad del paciente, analizada a partir de los diagnósticos.

El diagnóstico puede ser simple o único, y puede ser múltiple, esto es, al paciente le han sido diagnosticadas varias enfermedades, aunque sólo una haya sido la causa de la hospitalización; de esta manera, el registro de alta hospitalaria recoge el denominado diagnóstico principal (causa de la hospitalización) y otros diagnósticos, que pueden pertenecer al mismo o a distintos capítulos de la CIE-9-MC (M. ${ }^{\circ}$ de Sanidad y Consumo, 1994).

El número de diagnósticos varía de 1 a 7 , alcanzando una media de 2,1 diagnósticos por persona, con una desviación típica de 1,4 (Cuadro V). El 43,2 \% de la población tiene un sólo diagnóstico (o diagnóstico principal), el $27 \%$ tiene 2 , y casi un tercio de la población que ha causado alta hospitalaria supera el número medio

CUADRo V

NÚMERO DE DIAGNÓSTICOS DE LA POBLACIÓN

QUE HA CAUSADO ALTA HOSPITALARIA (1994)

(EN \% SOBRE N)

\begin{tabular}{|c|c|c|c|c|c|}
\hline \multirow{2}{*}{$\begin{array}{c}\text { Núm. de } \\
\text { diagnósticos }\end{array}$} & \multicolumn{3}{|c|}{ Sexo } & \multicolumn{2}{|c|}{ Edad } \\
\hline & Varón & Mujer & Total & 0-64 años & $65+a \tilde{o} o s$ \\
\hline 1 diagnóstico .... & 43,6 & 42,6 & 43,2 & 47,5 & 33,4 \\
\hline $2 \ldots \ldots \ldots \ldots \ldots \ldots$ & 23,5 & 30,1 & 27,0 & 29,4 & 21,7 \\
\hline $3 \ldots \ldots \ldots \ldots \ldots$ & 13,7 & 13,0 & 13,3 & 11,8 & 16,7 \\
\hline $4 \quad \ldots \ldots \ldots \ldots \ldots \ldots \ldots$ & 10,5 & 8,3 & 9,3 & 6,8 & 15,0 \\
\hline $5 \quad \ldots \ldots \ldots \ldots \ldots \ldots$ & 5,1 & 3,7 & 4,3 & 2,8 & 7,6 \\
\hline $6 \ldots \ldots \ldots \ldots \ldots \ldots$ & 1,9 & 1,3 & 1,6 & 1,0 & 3,0 \\
\hline $7 \ldots \ldots \ldots \ldots \ldots$ & 1,7 & 1,0 & 1,3 & 0,7 & 2,6 \\
\hline $\mathrm{N}$ & 1.190 .461 & 1.340 .904 & 2.531 .365 & 1.742 .141 & 791.974 \\
\hline Media ............... & 2,2 & 2,1 & 2,1 & 1,9 & 2,6 \\
\hline
\end{tabular}


de diagnósticos, esto es, tiene diagnosticadas tres o más patologías ${ }^{4}$.

Esta distribución varía si se tiene en cuenta el sexo y la edad del hospitalizado; son los varones, de una parte, y las personas con $65 \mathrm{y}$ más años, de otra, quienes alcanzan un mayor número de diagnósticos. Así, los varones llegan a 2,2 de media (2,1 entre las mujeres) y los ancianos a 2,6 (frente a 1,9 de la población menor de 65 años).

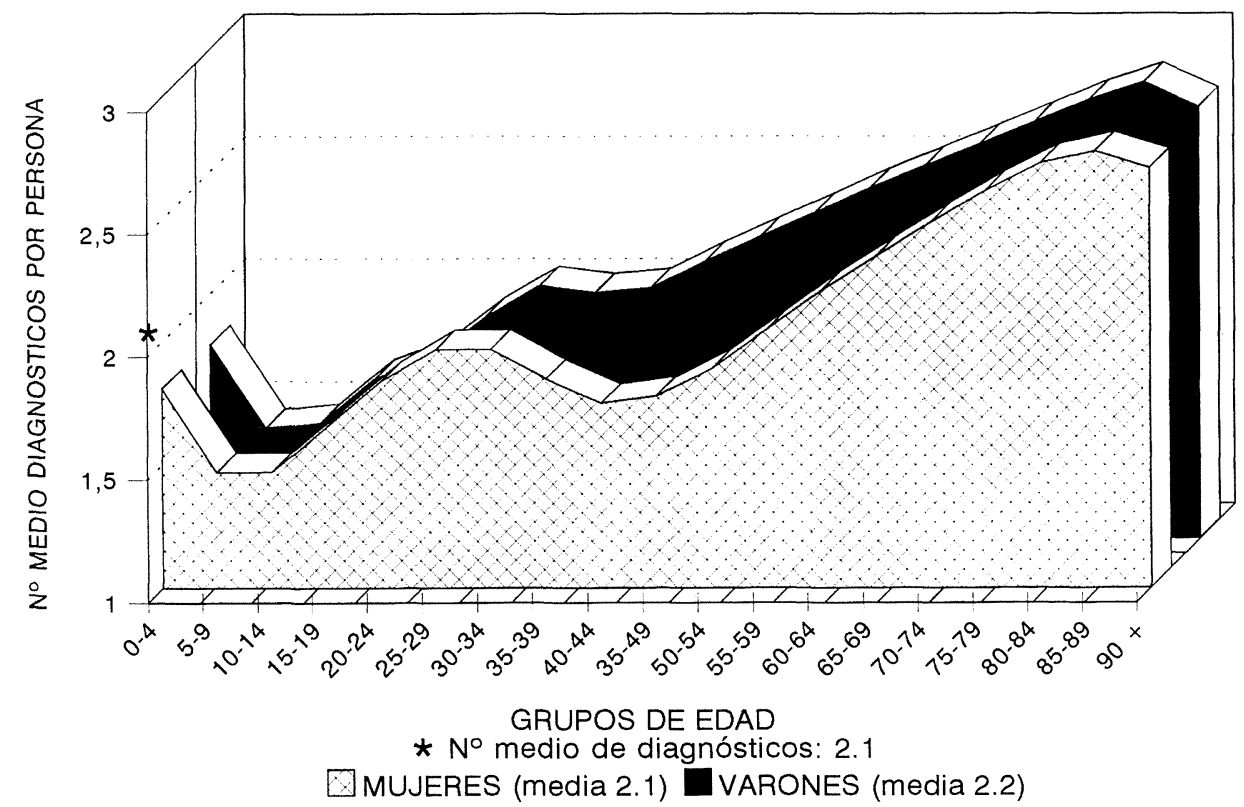

Figura 3.-Número medio de diagnósticos de la población que ha causado alta hospitalaria (1994) por grupos de edad y sexo.

Si se desciende en la edad, son las mujeres las que mayor número de diagnósticos presentan hasta los 9 años, y entre los $20 \mathrm{y}$ los 29; en éste último caso, de nuevo, el aspecto de la maternidad

4 Los modelos de morbilidad difieren entre admisiones hospitalarias, médicos generales y encuestas de salud (Davies, 1985). Así, la Encuesta Nacional de Salud de 1993 muestra que un $70 \%$ de la población declaraba no padecer ninguna enfermedad, un 21 \% informaba un diagnóstico y sólo un 9 \% padecía dos o más (Abellán et al., 1996). 
podría ser el factor explicativo; entre los 10 y los 19 años no ha diferencias por sexo en el valor medio de patologías diagnosticadas, mientras que en el resto de las edades predominan los varones (Figura 3).

Se observa una tendencia clara a aumentar la duración de la estancia a medida que lo hace el número de diagnósticos (Cuadro VI), de manera que un $73 \%$ de la población con una sola enfermedad diagnosticada permanece en hospital menos de una semana frente a tan sólo un $33 \%$ de quienes tienen más de 5 enfermedades.

\section{CuAdro VI}

NÚMERO DE DIAGNÓSTICOS POR DÍAS DE ESTANCIA EN HOSPITAL DE LA POBLACIÓN QUE HA CAUSADO ALTA HOSPITALARIA (1994)

\begin{tabular}{|c|c|c|c|c|c|c|c|}
\hline \multirow{2}{*}{$\begin{array}{l}\text { Días de } \\
\text { estancia }\end{array}$} & \multicolumn{7}{|c|}{ Núm. de diagnósticos } \\
\hline & 1 & 2 & 3 & 4 & $5+$ & Total & $\begin{array}{l}\text { Núm. } \\
\text { medio }\end{array}$ \\
\hline 0 días .... & 4,2 & 2,9 & 1,7 & 1,1 & 1,0 & 2,9 & 1,6 \\
\hline $1-7 \ldots \ldots \ldots$ & 69,1 & 65,6 & 51,7 & 40,4 & 31,8 & 60,5 & 1,9 \\
\hline $8-14 \ldots \ldots$ & 17,1 & 19,5 & 27,2 & 30,8 & 31,0 & 21,4 & 2,5 \\
\hline $15-21 \ldots$. & 5,2 & 6,3 & 9,8 & 13,2 & 15,2 & 7,6 & 2,7 \\
\hline $22-31 \ldots$. & 2,6 & 3,3 & 5,4 & 7,8 & 10,1 & 4,2 & 2,9 \\
\hline $32+\ldots \ldots$ & 1,8 & 2,3 & 4,2 & 6,8 & 10,9 & 3,4 & 3,2 \\
\hline$\%$ Total ... & 43,2 & 27,0 & 13,3 & 9,3 & 7,2 & 100,0 & \\
\hline $\mathrm{N}$ & 1.092 .580 & 684.193 & 337.515 & 236.564 & 183.230 & 2.534 .082 & 2,1 \\
\hline
\end{tabular}

\section{La morbilidad hospitalaria}

Según el cuadro general de diagnósticos, casi dos tercios de la población que ha sido dada de alta en los hospitales españoles tienen 
como diagnóstico principal enfermedades que se engloban en seis grandes tipos ${ }^{5}$ (Cuadro VII).

El diagnóstico más representativo es el relacionado con las complicaciones del embarazo, parto y puerperio, que alcanza al $13,1 \%$ de la población; es un capítulo que atañe al sexo femenino y constituye un motivo importante de ocupación de camas en los servicios de ginecología y obstetricia, afectando a un 43,2 \% de la población entre 16 y 39 años; se trata de un diagnóstico propio del período fértil, de manera que la población así diagnosticada alcanza una edad media de 29,3 anos con una desviación típica de 5,3.

El segundo diagnóstico más frecuente, que se corresponde con las enfermedades del aparato digestivo (13\%), se produce con una media de edad de 50 años y una desviación de 25, observándose un mayor predominio de varones y una tendencia equilibrada por edad, aunque con mayor incidencia a partir de los 40 años.

Las enfermedades relacionadas con el aparato circulatorio (11,3\%) son también predominantes entre los varones. Aunque la incidencia de las mismas comienza a manifestarse de forma muy significativa a partir de los 40 años, es el diagnóstico hospitalario de mayor prevalencia en la tercera edad, por lo que la edad media de la población diagnosticada con este tipo de enfermedades es elevada (66 años con una desviación de 15,7).

Las enfermedades respiratorias (8,7\%) afectan a casi una quinta parte de la población infantil (entre 0 y 15 años) y a una décima parte de los ancianos; son también los varones los más desfavorecidos por este diagnóstico.

La incidencia de tumores (8,3\%) es mayor entre los hombres; comienza a manifestarse con frecuencias altas a partir de los 40 años y alcanza a un $11,8 \%$ de los viejos, de ahí que la edad media a la que se producen sea también elevada ( 58,7 años).

Las lesiones y envenenamientos (8,3\%) son especialmente impor-

5 Tanto el C.M.B.D. de 1994 como la Encuesta de Morbilidad Hospitalaria de 1991 (I.N.E.) muestran que más de un tercio de la población que ha causado alta hospitalaria ha sido diagnosticada bajo uno de los cuatro epígrafes de mayor incidencia: enfermedades del aparato digestivo, enfermedades del aparato circulatorio, del aparato respiratorio y las lesiones y envenenamientos. Se exceptúa aquí el diagnóstico 11: complicaciones con el embarazo, parto y puerperio, ya que el C.M.B.D. de 1994 engloba el parto normal en el grupo V y en la E.M.H. constituye un grupo independiente. 
CUADRo VII

DIAGNÓSTICO PRINCIPAL DE LA POBLACIÓN QUE HA CAUSADO ALTA HOSPITALARIA (1994), SEGÚN GRUPOS DE EDAD, SEXO Y EDAD MEDIA (EN \% SOBRE N)

\begin{tabular}{|c|c|c|c|c|c|c|c|c|c|c|c|}
\hline \multirow{2}{*}{$\begin{array}{c}\text { Diagnóstico } \\
\text { principal } \\
\text { (capitulos de la }^{\text {CIE-9-MC) }}{ }^{3} \\
\end{array}$} & \multicolumn{7}{|c|}{ Edad } & \multicolumn{2}{|c|}{ Sexo } & \multicolumn{2}{|c|}{ Edad } \\
\hline & $0-1$ & $2-15$ & $16-39$ & $40-64$ & $65-74$ & $75+$ & Conjunto & Varón & Mujer & Media & $\begin{array}{l}\text { Desv } \\
\text { típica }\end{array}$ \\
\hline $\begin{array}{l}\text { 1. Enf. infecciosas } \\
\text { y parasitarias . ..... }\end{array}$ & 8,0 & 5,6 & 3,0 & 1,6 & 1,1 & 1,2 & 2,6 & 3,3 & 1,9 & 30,6 & 25,3 \\
\hline $\begin{array}{l}\text { 2. Neoplasias .... } \\
\text { 3. Enf. endocrinas }\end{array}$ & 0,5 & 2,8 & 3,1 & 13,0 & 13,2 & 10,3 & 8,3 & 9,1 & 7,5 & 58,7 & 18,4 \\
\hline $\begin{array}{l}\text { nutritivas y } \ldots \ldots \ldots \\
\text { 4. Enf. de la san. }\end{array}$ & 0,8 & 1,7 & 1,0 & 2,0 & 1,9 & 2,1 & 1,7 & 1,4 & 1,9 & 52,2 & 24,3 \\
\hline $\begin{array}{l}\text { gre y } \ldots \ldots \ldots \ldots \ldots \\
5 \text {. Trastornos men- }\end{array}$ & 0,4 & 1,4 & 0,4 & 0,7 & 1,0 & 1,5 & 0,8 & 0,8 & 0,9 & 53,9 & 27,6 \\
\hline $\begin{array}{l}\text { tales } \ldots \ldots \ldots \ldots \ldots \\
\text { 6. Enf. sistema ner- }\end{array}$ & 0,2 & 0,6 & 2,9 & 2,1 & 0,9 & 0,9 & 1,7 & 2,1 & 1,4 & 42,8 & 19,3 \\
\hline $\begin{array}{l}\text { vioso y de } \ldots \ldots \ldots \\
\text { 7. Enf. sistema cri- }\end{array}$ & 2,4 & 6,8 & 2,7 & 6,6 & 9,7 & 9,9 & 6,3 & 6,4 & 6,2 & 56,0 & 24,2 \\
\hline $\begin{array}{l}\text { culatorio .............. } \\
\text { 8. Enf. aparato res- }\end{array}$ & 0,6 & 0,7 & 2,3 & 14,2 & 20,5 & 23,4 & 11,3 & 13,8 & 9,1 & 65,8 & 15,7 \\
\hline $\begin{array}{l}\text { piratorio } \ldots \ldots \ldots \ldots \\
9 \text {. Enf. aparato di- }\end{array}$ & 13,6 & 20,5 & 4,3 & 6,1 & 9,6 & 12,5 & 8,7 & 11,8 & 5,9 & 45,5 & 30,8 \\
\hline $\begin{array}{l}\text { gestivo ................. } \\
\text { 10. Enf. aparato gé- }\end{array}$ & 9,4 & 15,0 & 8,5 & 16,4 & 14,6 & 14,0 & 13,0 & 16,1 & 10,2 & 49,9 & 24,9 \\
\hline $\begin{array}{l}\text { nito-urinario ...... } \\
\text { 11. Complicaciones }\end{array}$ & 3,7 & 6,8 & 5,5 & 10,4 & 7,6 & 5,7 & 7,2 & 6,2 & 8,1 & 48,7 & 22,6 \\
\hline $\begin{array}{l}\text { del embarazo, ..... } \\
\text { 12. Enf. de la piel y }\end{array}$ & 0,0 & 0,2 & 43,2 & 1,5 & 0,0 & 0,0 & 13,1 & 0,0 & 24,7 & 29,3 & 5,3 \\
\hline $\begin{array}{l}\text { del } \ldots \ldots \ldots \ldots \\
\text { 13. Enf. sistema os- }\end{array}$ & 0,8 & 1,5 & 2,0 & 0,9 & 0,7 & 0,7 & 1,2 & 1,6 & 0,9 & 37,2 & 23,4 \\
\hline $\begin{array}{l}\text { teo-mioarticular y .. } \\
\text { 14. Anomalías con- }\end{array}$ & 0,4 & 3,4 & 4,3 & 7,1 & 5,3 & 2,1 & 4,6 & 4,7 & 4,5 & 48,2 & 20,2 \\
\hline $\begin{array}{l}\text { génitas } \ldots \ldots \ldots \ldots \\
\text { 15. Enf. con origen }\end{array}$ & 6,1 & 5,4 & 0,7 & 0,3 & 0,2 & 0,1 & 1,1 & 1,4 & 0,8 & 14,5 & 18,7 \\
\hline $\begin{array}{l}\text { en el periodo peri- } \\
\text { natal ............. } \\
\text { 16. Sintomas, mal }\end{array}$ & 34,2 & 0,3 & 0,1 & 0,0 & 0,0 & 0,0 & 1,6 & 1,7 & 1,3 & 0,5 & 4,4 \\
\hline definidos ............ & 5,9 & 8,5 & 3,1 & 4,7 & 4,1 & 4,0 & 4,4 & 5,0 & 3,9 & 43,5 & 27,1 \\
\hline
\end{tabular}


CUADro VII (Continuación)

DIAGNÓSTICO PRINCIPAL DE LA POBLACIÓN QUE HA CAUSADO ALTA HOSPITALARIA (1994), SEGÚN GRUPOS DE EDAD, SEXO Y EDAD MEDIA (EN \% SOBRE N)

\begin{tabular}{|c|c|c|c|c|c|c|c|c|c|c|c|}
\hline \multirow{2}{*}{$\begin{array}{c}\text { Diagnóstico } \\
\text { principal } \\
\text { (capítulos de la }^{\text {CIE-9-MC) }}{ }^{3}\end{array}$} & \multicolumn{7}{|c|}{ Edad } & \multicolumn{2}{|c|}{ Sexo } & \multicolumn{2}{|c|}{ Edad } \\
\hline & $0-1$ & $2-15$ & $16-39$ & $40-64$ & $65-74$ & $75+$ & Conjunto & Varón & Mujer & Media & $\begin{array}{l}\text { Desv. } \\
\text { típica }\end{array}$ \\
\hline \multirow{3}{*}{$\begin{array}{l}\text { 17. Lesiones y .... } \\
\text { V. Clasificación su- } \\
\text { plementaria de fac- } \\
\text { tores que .......... } \\
\text { E. Clasificación su- } \\
\text { plementaria de cau- } \\
\text { sas externas ........ }\end{array}$} & 2,6 & 14,2 & 8,9 & 7,4 & 6,1 & 9,7 & 8,3 & 10,2 & 6,6 & 44,6 & 26,6 \\
\hline & 10,4 & 4,3 & 4,2 & 5,0 & 3,6 & 1,8 & 4,3 & 4,3 & 4,2 & 40,3 & 24,6 \\
\hline & 0,0 & 0,0 & 0,0 & 0,0 & 0,0 & 0,0 & 0,0 & 0,0 & 0,0 & 40,6 & 23,7 \\
\hline $\mathrm{N} \ldots \ldots \ldots \ldots \ldots$ & 116.530 & 217.985 & 740.956 & 666.577 & 409.491 & 382.406 & 2.533 .941 & 1.190 .409 & 1.340 .819 & 46,6 & 25,2 \\
\hline
\end{tabular}


tantes entre los 2 y los 15 años, así como entre los adultos jóvenes y en los mayores. Su incidencia es, de nuevo, fundamental en los varones.

Con una incidencia menos relevante las enfermedades del aparato genito-urinario $(7,2 \%)$ afectan sobre todo al sexo femenino en las edades adultas y viejas.

Las enfermedades del sistema nervioso y de los órganos de los sentidos $(6,3 \%)$ apenas muestran diferencias por sexo pero aumentan con la edad, en referencia a la incidencia del deterioro sensorial a medida que se envejece (por ejemplo, discapacidad visual y auditiva). La misma tendencia se observa para las enfermedades del sistema osteomioarticular y tejido conectivo (4,6 \%).

El resto de los diagnósticos tienen frecuencias menores al $5 \%$ en su incidencia. Dentro de ellas merecen ser destacadas las enfermedades infecciosas y parasitarias, las anomalias congénitas y las enfermedades con origen en el periodo perinatal, todas ellas asociadas con una edad media inferior a la del conjunto de la población que ha causado alta hospitalaria puesto que se producen, básicamente, entre la población más joven e infantil.

La incidencia de los trastornos mentales como morbilidad hospitalaria es muy baja, afectando al 1,7 \% de la población española que ha causado alta hospitalaria en 1994, y con una mayor incidencia entre los 16 y 64 años ${ }^{6}$.

Las enfermedades endocrinas, nutritivas y metabólicas y trastornos de la inmunidad, y las enfermedades de la sangre y de los órganos hematopoyéticos afectan, en su conjunto, al 2,5\% de la población.

Poco más del 4,4\% de las altas hospitalarias tenían como diagnóstico principal sintomas, signos y estados mal definidos.

En definitiva, y exceptuando la mayor proporción de altas hospitalarias debidas a la maternidad (Olivera, 1996), puede observarse (Figura 4) que, entre los seis diagnósticos principales más frecuentes, la incidencia de enfermedades digestivas, circulatorias y respiratorias varía, sobre todo, en función de la edad, con un pico muy claro entre los viejos para las circulatorias (Castello et al.; 1988; Sánchez,

6 En Francia la Enquetê nationale sur les hospitalisés 1991-1992 cifra en un 21,9\% el porcentaje de problemas mentales sobre los diagnósticos al ingreso hospitalario (Com-Ruelle, 1995). 

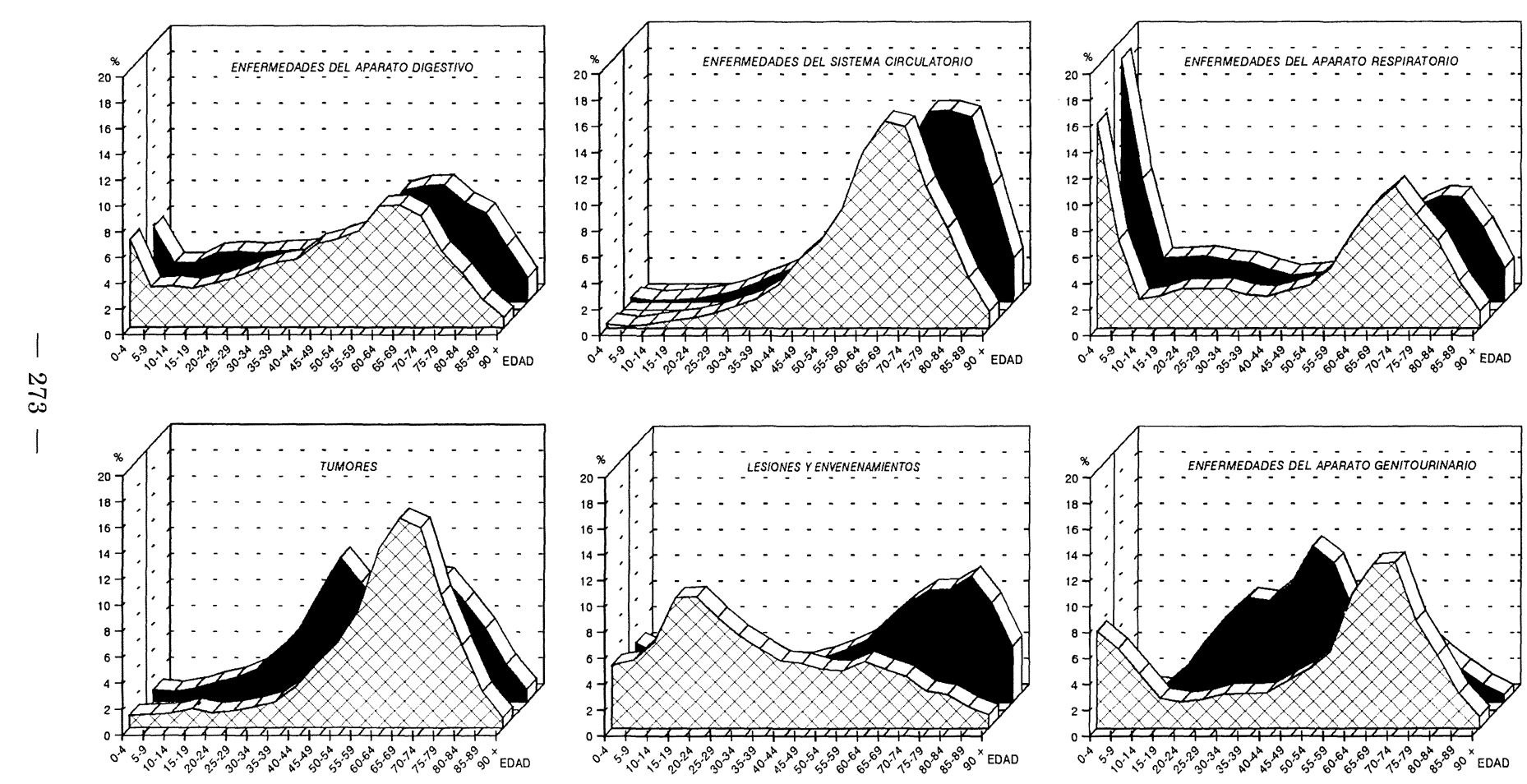

Figura 4.-Diagnósticos principales más frecuentes de la población que ha causado alta hospitalaria (1994), por edad y sexo (en trama varones, en negro mujeres). 
1990), y dos resaltes que coinciden con las edades más tempranas y con las más avanzadas tanto en las digestivas como en las respiratorias, posiblemente debido a diferentes procesos agudos o crónicos. Por su parte, los tumores, lesiones y enfermedades genitourinarias presentan una variación en su incidencia más evidente según el sexo; las neoplasias son más precoces entre las mujeres (cáncer de mama, cáncer de útero, ...) (Caicoya, 1996) y algo más tardías entre los hombres (cáncer de pulmón, de próstata, ...); los varones son más proclives a lesionarse a edades jóvenes y las mujeres en la vejez; y los problemas genitourinarios corresponden en el sexo femenino con su periodo fértil y el inicio de la menopausia.

Sin embargo, no son las enfermedades de mayor incidencia las que causan estancias hospitalarias más largas ni las que generan mayor número de patologías, de manera que son los trastornos mentales las enfermedades que requieren más días de estancia y, en el lado opuesto, las estancias más cortas son las causadas por las complica-

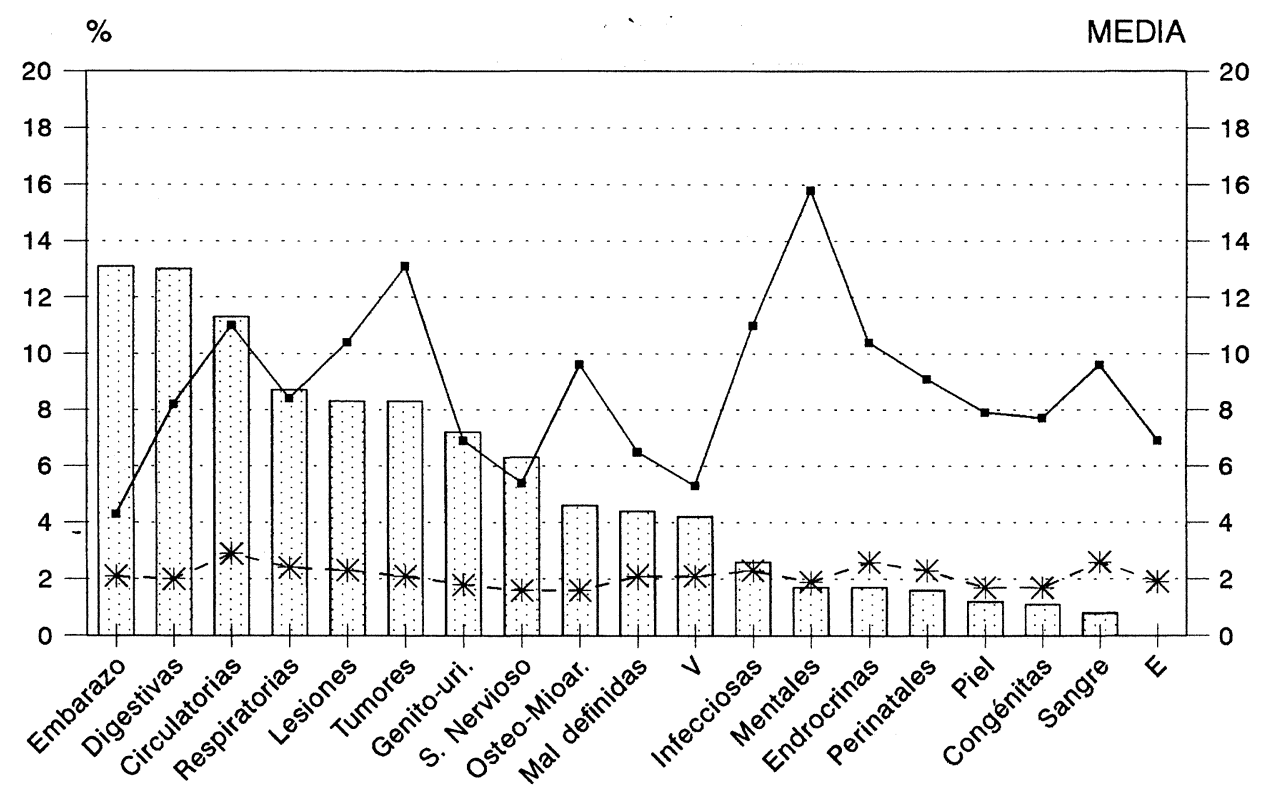

DDIAGNOSTICO PRINCIPAL - No MEDIO DIAS ESTANCIA * No MEDIO PATOLOGIAS

Figura 5.-Diagnóstico principal por número medio de dias de estancia y de patologías de la población que ha causado alta hospitalaria (1994). 
ciones con el embarazo, parto y puerperio que alcanzan 4,3 días de estancia media aunque sea el diagnóstico más frecuente entre la población (Figura 5).

Puesto que el $62 \%$ de la población ingresa en hospital vía urgencia y el $93 \%$ causa alta con regreso a su domicilio estas dos variables no registran diferencias en la frecuencia de diagnósticos con respecto al conjunto de las altas hospitalarias. Sin embargo, el acceso programado a hospital introduce variaciones, en tanto que entre los cinco diagnósticos más frecuentes se encuentran los tumores, las enfermedades del aparato digestivo, del sistema nervioso y de los órganos de los sentidos, del aparato genito-urinario y las enfermedades del sistema osteo-mioarticular y tejido conectivo (Cuadro VIII).

Por su lado, la muerte como circunstancia del alta hospitalaria se relaciona, en primer lugar, a enfermedades del sistema circulatorio (Banchs, 1990) (que explican más de una quinta parte de las altas hospitalarias por este motivo), seguidas muy de cerca por los tumores; las enfermedades del aparato respiratorio y las del aparato digestivo generan, conjuntamente, el $23 \%$ de las muertes como causa del alta hospitalaria. Se trata, en buena medida, de los diagnósticos que más incidencia tienen entre la población española que ha causado alta hospitalaria, así como las causas más frecuentes de mortalidad (Gómez, 1990).

Los diagnósticos asociados a la muerte como circunstancia del alta hospitalaria entre los mayores siguen la misma tendencia que entre la población en su conjunto, siendo las enfermedades relacionadas con el sistema circulatorio las más letales, al igual que ocurre entre la población femenina a todas las edades. Sin embargo, es el cáncer el primer diagnóstico de muerte entre los varones y también entre la población no anciana, en tanto que las enfermedades del aparato respiratorio ocupan el tercer lugar entre la población mayor. Asimismo por edad, se observa una diferencia muy acusada en las muertes causadas por enfermedades infecciosas y parasitarias como diagnóstico principal que, si bien alcanzan al $9 \%$ de la población hasta los 64 años, no llega al $2 \%$ de los mayores de 65 . Es preciso señalar, además, que casi un $6 \%$ de las mujeres que causan alta por muerte tienen asociado como diagnóstico principal las complicaciones con el embarazo, parto y puerperio.

Por otro lado, se observa que más de tres cuartas partes de los 
CUADRO VIII

DISGNÓSTICO PRINCIPAL MÁS FRECUENTE DE LA POBLACIÓN QUE HA CAUSADO ALTA HOSPITALARIA (1994) SEGÚN TIPO

DE INGRESO Y CIRCUNSTANCIAS DEL ALTA, POR EDAD

Y SEXO (EN \% SOBRE N)

\begin{tabular}{|c|c|c|c|c|c|}
\hline \multicolumn{6}{|c|}{ INGRESO PROGRAMADO $(38,4 \%)$} \\
\hline Diagnóstico principal $^{3}$ & Varón & Mujer & $\begin{array}{l}0-64 \\
\text { años }\end{array}$ & 65+años & Total \\
\hline 2. Neoplasias...$\ldots \ldots \ldots \ldots \ldots \ldots$ & 12,5 & 3,4 & 11,4 & 16,7 & 12,9 \\
\hline 6. Enf. sistema nervioso ......... & 11,5 & 13,3 & 8,1 & 22,9 & 12,4 \\
\hline 7. Enf. sistema circulatorio ..... & 9,2 & 6,3 & 6,5 & 10,8 & 7,7 \\
\hline 8. Enf. aparato respiratorio ..... & 8,0 & 4,6 & 7,4 & 3,9 & 6,3 \\
\hline 9. Enf. aparato digestivo .......... & 15,6 & 10,1 & 13,2 & 11,8 & 12,8 \\
\hline 10. Enf. aparato genito-urinario .. & 9,3 & 11,6 & 10,4 & 10,5 & 10,4 \\
\hline 13. Enf. sistema ósteo-mioarticu- & & & & & \\
\hline $\operatorname{lar} \ldots \ldots \ldots \ldots \ldots \ldots \ldots \ldots \ldots \ldots \ldots \ldots$ & 8,8 & 9,9 & 9,9 & 8,1 & 9,4 \\
\hline Total ..................... & 66,9 & 54,6 & 59,5 & 80,8 & 65,6 \\
\hline $\mathrm{N}$ del grupo .................... & 482.005 & 487.889 & 687.193 & 283.542 & 970.735 \\
\hline
\end{tabular}

ALTA POR MUERTE (2,8\%)

\begin{tabular}{|c|c|c|c|c|c|}
\hline Diagnóstico principal ${ }^{3}$ & Varón & Mujer & $\begin{array}{l}0-64 \\
\text { años }\end{array}$ & 65+años & Total \\
\hline 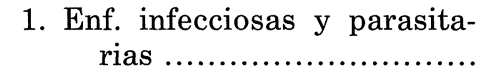 & 5,0 & 3,0 & 9,0 & 1,9 & 4,2 \\
\hline 2. Neoplasias ...................... & 26,1 & 19,7 & 27,6 & 21,3 & 23,3 \\
\hline 7. Enf. sistema circulatorio ..... & 24,1 & 29,0 & 14,0 & 31,8 & 26,2 \\
\hline 8. Enf. aparato respiratorio ..... & 14,1 & 9,6 & 6,7 & 14,6 & 12,1 \\
\hline 9. Enf. aparato digestivo ......... & 11,1 & 10,7 & 10,0 & 11,4 & 10,9 \\
\hline 11. Complicaciones del embara- & & & & & \\
\hline zo, parto y puerperio ........ & 0,0 & 5,6 & 7,7 & 0,0 & 2,4 \\
\hline Total .................... & 80,4 & 77,6 & 75,0 & 81,0 & 79,1 \\
\hline $\mathrm{N}$ del grupo .... & 40.102 & 30.473 & 22.275 & 48.352 & 70.627 \\
\hline
\end{tabular}


pacientes que causan alta hospitalaria por muerte tenían diagnosticadas al menos dos patologías o, más aún, cerca del $25 \%$ de estos pacientes tenían cinco o más patologías, tendencia más acusada en el caso de los varones y también de los ancianos.

\section{Conclusiones}

La morbilidad hospitalaria en España está en la línea de los resultados observados en otros países desarrollados en el sentido de que las mujeres se hospitalizan más que los varones (Davies, 1985; Sorty-le-Guellec, 1993), si bien algunos estudios realizados en España utilizando la encuesta de morbilidad del I.N.E. apuntan lo contrario (Olivera, 1996).

La estructura por edad de la población que ha causado alta hospitalaria es muy diferente a la de la población española en su conjunto, poniéndose de manifiesto la fuerte presencia de los mayores en hospital y las tasas de hospitalización más bajas entre los no ancianos. Además, las personas que más tiempo permanecen en hospital son las de mayor edad, que se corresponden, asimismo, con las que tienen diagnosticadas más número de patologías.

La mayor parte de los ingresos hospitalarios se realizan vía urgencias, lo que llevaría a replantearse el sistema de referencia y las listas de espera.

La incidencia de enfermedades es también diferente según el sexo, resaltando las mujeres, casi exclusivamente, en las complicaciones con el embarazo, parto y puerperio, y en las del aparato genitourinario. Por edad y a grandes rasgos se perfilan dos subgrupos; por un lado, los menores de 15 años que registran mayor incidencia de enfermedades relacionadas con el período perinatal, aparato respiratorio y digestivo, lesiones e infecciosas; y por otro, los mayores de 40 años con enfermedades del sistema circulatorio, tumores y digestivas, en un modelo que podría relacionarse con patologías asociadas con problemas agudos, en el primer caso, y con problemas crónicos y degenerativos, en el segundo.

Dentro de este modelo resaltaría un «valle de salud» entre los 16 y 39 años cuyos principales problemas se asocian con el factor maternidad. El predominio de mujeres hospitalizadas sólo durante su 
edad fértil y a partir de los 80 años corrobora la imagen de salud atribuida tradicionalmente al sexo femenino, al sufrir menos incidencia de enfermedades cuya gravedad deriva a la atención hospitalaria, menor número de patologías y estancias hospitalarias más cortas.

Si bien la mayor parte de las altas hospitalarias suceden con el regreso al domicilio, un $1 \%$ de las personas que causan alta con $65 \mathrm{y}$ más años son transferidas a otro centro sociosanitario. Este hecho, a la luz del progresivo envejecimiento de la población, unido al descenso de los casos fatales y a la mejora de la supervivencia, lleva a replantearse la capacidad de la sociedad para ofrecer soluciones alternativas a la institución hospitalaria en los casos oportunos.

\section{BIBLIOGRAFÍA}

Abellán García, A.; Fdez.-Mayoralas Fernández, G.; Rodríguez Rodriguez, V., y Rojo PÉREZ, F.: «La valoración del estado de salud: morbilidad y autopercepción», Revista de Gerontología, núm. 4, 1992, pp. 231-236.

-: Envejecer en España. Manual estadístico sobre el envejecimiento de la población, Madrid, 1996, Fundación Caja de Madrid, 74 p.

Andrews, F. M.: «Social indicators and health-for-all», Social Science and Medicine, vol 15 C, 1981, pp. 219-223.

BANChS Galtes, L.: «Factores de riesgo de la enfermedad coronaria. Proyecto de perfil multivariante para su uso por el profesional de la medicina», Medicina Integral. $\mathrm{Me}$ dicina preventiva y asistencial, vol. 15, núm. 9, pp. 348-358, 1990.

CAIcoya, M.: «Aspectos actuales de la prevención primaria del cáncer», Revista de Gerontología, vol. 6 , núm. 1, pp. 8-18, 1996.

Castello, R.; Alegría, E.; Merino, A., et al.: «Evolución a medio plazo de los pacientes mayores de sesenta y cinco años de infarto agudo de miocardio», Revista Española de Cardiología, vol. 41, pp. 78-82, 1988.

Com-Ruelle, L.: Les étapes diagnostiques et la maladie principale des hospitalisés en 1992, París, 1995, CREDES, núm. 1.052, 150 p.

Com-Ruelle, L., y Sourty-le-Guellec, M. J.: Enquête sur les hospitalisés. Morbidité hospitalière. Associations de diagnostique au cours d'une hospitalisation. Les hospitalisés sortants, París, 1989, CREDES, núm. 811, 158 p.

Com-Ruelle, L., y Dumesnil, S.: Les associations de diagnostics et les indicateurs de morbidité des hospitalisés en 1992, París, 1995, CREDES, núm. 1.073, 186 p.

DAVIES, A. N.: «Epidemiology and the challenge of ageing», International Journal of Epidemiology, vol 14, núm. 1, pp. 9-19, 1985.

Fernández.-Mayoralas Fernández, G.; Abellán Garcia, A.; Rodríguez Rodríguez, V., y Rojo PÉrez, F.: «Desigualdades en salud y envejecimiento demográfico», Anales de Geografia de la Universidad Complutense, núm. 13, 1993, pp. 139-154.

Fernández-Mayoralas Fernández, G.; Rodriguez Rodrfguez, V.; Rojo Pérez, F., y Barrios Álvarez, L.: La utilización de los servicios sanitarios a partir de la Encuesta Nacional de Salud de 1993, Madrid, 1995, Investigación encargada por el M. de Sanidad y Consumo, 74 p. + anexos (mecanografiado). 
Gómez Redondo, R.: Las causas de muerte en España, 1981-1985. Análisis diferencial por sexo y edad. Madrid, 1990, Instituto de Demografía, CSIC, Documento núm. 4, 94 p.

Hansluwka, H. E.: «Measuring the health of populations, indicators and interpretations», Social Science and Medicine, vol. 20, núm. 12, 1985, pp. 1207-1224.

Hulka, B. S., y WheAt, J. R.: «Patterns of utilization: the patient perspective», Medical Care, vol. 23, núm. 5, 1985, pp. 438-460.

KäKKINEN, U.: «The production of health and the demand for health care in Finland», Social Science and Medicine, vol. 33, núm. 3, 1991, pp. 225-237.

Keskimaki, I.; Salinto, M., y Aro, S.: «Socioeconomic equity in Finnish hospital care in relation to need», Social Science and Medicine, vol. 41, núm. 3, 1995, pp. 425-431.

LinN, B. S., y LinN, M. W.: «Objective and self assessed health in the old and very old», Social Science and Medicine, vol. 14 A, 1980, pp. 311-315.

Ministerio de SANidad y Consumo: Clasificación Internacional de Enfermedades. 9. ${ }^{a}$ Revisión. Modificación Clinica. Vol 1: Enfermedades; lista tabular, Madrid, 1994, M.S.C., $1.158 \mathrm{p}$.

Newbold, K. B.; EYles, J., y BIRCH, S.: «Equity in health care: methodological contributions to the analysis of hospital utilization within Canada», Social Science and Medicine, vol. 40, núm. 9, 1995, pp. 1181-1192.

Olivera Poll, A.: «Población, salud y bienestar», Treballs de la Societat Catalana de Geografia, núm. 41, 1996, pp. 135-171.

Rojo Pérez, F., y Fdez.-Mayoralas Fernández, G.: «El Conjunto Mínimo Básico de Datos (CMBD) como fuente de estudio para la morbilidad hospitalaria», Revista Estudios Geográficos, vol. LVII, núm. 225, 1996, pp. 738-747.

-: "Condiciones sociosanitarias de las personas de edad en España», en Abellan GAR CfA, A. (ed.): Una España que envejece, Sevilla, 1992, Universidad Hispanoamericana Santa María de la Rábida, pp. 97-123.

SAnchez, R. G.: «Hacia un estudio epidemiológico sobre factores de riesgo cardiovasculares en el anciano en España», Revista Española de Geriatría y Gerontologia, vol. 25, núm. 6, 1990, pp. 383-388.

Sermet, C.: Les personnes agées à l'hôpital. France 1981-1982, París, 1987, CREDES, núm. $731,250 \mathrm{p}$.

Sourty-Le Guellec, M. J.: Enquête sur les hospitalisés. Caracteres socio-demographiques, mouvements, durées de séjour. Tome 1: Les sortants, París, 1986, CREDES, núm. 697, $126 \mathrm{p}$.

-: Enquête sur les hospitalisés 1991-1992. Qui sont les hospitalisés? Où sont-ils? Depuis combien de temps?, París, 1993, CREDES, núm. 996, 78 p.

WAN, T. T. H., y ODELL, B. G.: «Factors affecting the use of social and health services among the elderly», Ageing and Society, vol. 1, part. 1, 1981, pp. 95-115.

RESUMEN: La morbilidad hospitalaria de la población española. Se analiza la morbilidad de la población española, tomando como base de estudio los hospitales del país recogidos en el C.M.B.D. (Conjunto Mínimo Básico de Datos) para 1994. Se descubre que la presencia en hospital está marcada por los niños hasta los dos años, las mujeres en edad fértil y los ancianos. Más de la mitad de las altas hospitalarias se asocian con el factor maternidad, así como con las enfermedades digestivas, circulatorias, respiratorias y los tumores. Las estancias más prolongadas se producen entre la población mayor que se corresponde, además, con el grupo demográfico que presenta mayor número de patologías diagnosticadas.

Palabras clave: Población, salud, morbilidad hospitalaria, España. 
ABstRaCT: Hospital morbidity of the Spanish population. The objective of this paper is to determine the hospital morbidity in Spain. The data are derived from the C.M.B.D. (the Minimum Basic Data Set for Hospital Statistics) (1994). The results indicate that infants, women in fertil periode and elderly people are the most consumers of hospital discharges. More than a half of the hospital discharges are related to the motherhood factor as well as to the digestive, cardiovascular and respiratory diseases and to the cancer. Elderly people are more likely to stay more days in hospital and this is the main demographic group in number of diagnosticated illness.

KEY woRDS: Population, health, hospital morbidity, Spain.

RÉSUmÉ: La morbidité hostipalière de la population espagnole. Cette étude s'intéresse par la morbidité hospitalière en Espagne. On utilise le C.M.B.D. (le minimum ensemble des donées basiquées) pour 1994. Le taux de présence en hospitalisation est plus élevé au sein des enfants de moins de 2 ans, des femmes en leur période fertile et des personnes âgées. Plus de la moitié des hospitalisés sortants est liée a la maternité, ainsi que aux malades de l'appareil digestif, circulatoire et respiratoire et aux tumeurs. Les durées de séjour sont supérieures chez les âgées qui présentent, en plus, des polypathologies.

Mots cLÉs: Population, santé, morbidité hospitalière, Espagne. 